\title{
TOXICITÉ DE DEUX MOLLUSCICIDES (BAYLUSCIDE ET FRESCON) A L'ÉGARD DE LA MOULE D'EAU DOUCE (Dreissena Polymorpha PALLAS)
}

\author{
par H. HOESTLANDT
}

(Laboratoire de Biologie Marine de I'Université Catholique de Lille 62 - Ambleteuse)

\section{1 - RESUME}

1.1 - Dans la lutte contre la moule d'eau douce, le Bayluscide est plus actif que le Frescon.

1.2 - Les Poissons semblent plus sensibles au Frescon quau Bayluscide; ils ne sont, en général, pas sensibles aux doses de Bayluscide utilisées pour détruire les moules

1.3 - Les Mammifères et l'Homme ne sont aucunement sensibles à ces Jeux molluscicides aux doses utilisées pour détruire les Mollusques; en effet, chez les Mammifères, les produits métaboliques formés sont éliminés en $24 \mathrm{~h}$.

1.4 - II est intéressant d'utiliser le Bayluscide en "solution» dans le Néoprène: dans ce cas, le molluscicide ne diffuse que très lentement dans l'eau ambiante (faible concentration dans l'eau, dissolution lente durant plusieurs mois).

1.5. - Quelques indications pratiques sont données pour l'utilisation du Bayluscide dans les eaux piscicoles et dans les canalisations d'alimentation en eau des villes ou des usines. 


\section{2 - INTRODUCTION}

La moule d'eau douce, ou moule zébrèe, Dreissena polymorpha PALLAS, qui est originaire de la Caspienne, est de plus en plus abondante dans les eaux douces courantes ou stagnantes de l'Europe Occidentale. Actuellement, il est fréquemment nécessaire de lutter contre l'invasion de cette moule pour deux raisons principales. Tout d'abord, cette moule est l'hôte intermédiaire du Trématode, Bucephalus polymorphus BAER. qui cause une mortalité importante des poissons d'eau douce. en particulier dans la Seine et ses affluents (KINKELIN, TUFFERY, LEYNAUD, ARRIGNON, 1968 a et b). D'autre part, cette moule envahit les canalisations d'alimentation en eau des villes ou des usines (Centrales Electriques, Usines Métallurgiques).

Antérieurement nous avons décrit une méthode thermique de destruction de ce mollusque qui ne résiste pas à une température de $+40 \circ \mathrm{C}$ durant 15 minutes (HOESTLANDT et LASSABLIERE, 1959). Cette méthode peut intéresser les Centrales Electriques ou les Usines Métallurgiques, car ces Industries éliminent généralement des eaux chaudes résiduaires. Nous avons également précisé la méthode d'utilisation du chlore pour les canalisations d'amenée d'eau dans les villes (HOESTLANDT, 1968, 1969), ce qui exige 10ppm de chlore résiduel et une action durant 12 heures. Selon certains auteurs (en particulier GREENSHIELDS and RIDLEY, 1957), la dose de 0,5ppm de chlore résiduel suffirait à la condition d'agir durant sept jours consécutifs.

La découverte relativement récente des molluscicides, en particulier dans la lutte contre les Gastéropodes tropicaux hôtes intermédiaires de la Bilharziose, nous a conduit à expérimenter sur un certain nombre de ces produits, dont deux d'entre eux méritent d'être retenus : le Bayluscide et le Frescon (HOESTLANDT, 197!).

\section{3 - ACTION TOXIQUE DU BAYLUSCIDE ET DU FRESCON}

\section{1 - Méthodes}

Le Bayluscide en poudre mouillable est un produit fabriqué par Bayer en Allemagne; c'est un sel d'éthanolamine du dichloro-2', 5 nitro-4' salicylanilide ; le Frescon mélangé à un solvant est un produit fabriqué par Shell en Angleterre, c'est le N-triphenylméthylmorpholine.

Les expériences effectuées en Laboratoire s'inspirent des méthodes mises au point par l'Organisation Mondiale de la Santé au cours de ses recherches sur la destruction des Molluscives vecteurs de la Bilharziose.

Les moules utilisées proviennent de la région de Thonon sur les bords du lac Léman (1); elles sont âgées de 2 à 3 ans. On les dispose par 20 ou par 30 dans des aquariums de verre. L'eau douce est déchlorée $(\mathrm{pH}=8,5)$. Les moules sont maintenues dans la solution toxique(1,5 litre) durant 24 heures à une température de $20 \circ \mathrm{C}$; la solution toxique est renouvelée après 12 heures. Les moules sont onsuite gardées en eau douce (renouvelée chaque jour) durant 9 jours, en effet les mortalités s'échelonnent sur 5 ou 6 jours. Le Bayluscide en solution dans l'eau est plus stable que le Ferscon; c'est pourquoi, seule la concentration des solutions de Frescon est contrôlée alu cours des essais par une méthode colorimétrique.

(1) Nous remercions Monsieur P. LAURENT, directeur de la Station Hydrobiologique de Thonon, qui nous a procuré ces moules 


\section{2 - Action sur les moules}

Les résultats des expériences sont donnés dans le tableau ci-apres et reportés sur le graphinut construit en données logarithmiques selon la méthode des probabilites transformées appelée "probit " (LITCHFIELD and WIL.COXON, 1942). La position de la droite de régression est calculée selon les méthodes statistiques habituelles.

\begin{tabular}{|c|c|c|c|c|c|}
\hline \multicolumn{3}{|c|}{ Action du Bayluscide } & \multicolumn{3}{|c|}{ Action du Frescon } \\
\hline $\begin{array}{c}\text { Concentration } \\
\text { en ppm }\end{array}$ & $\begin{array}{l}\text { Nombre } \\
\text { de morts }\end{array}$ & $\begin{array}{l}\text { Pourcentage } \\
\text { de mortalité }\end{array}$ & $\begin{array}{c}\text { Concentration } \\
\text { en ppm }\end{array}$ & $\begin{array}{l}\text { Nombre } \\
\text { de morts }\end{array}$ & $\begin{array}{l}\text { Pourcentage } \\
\text { de mortalité }\end{array}$ \\
\hline $\begin{array}{c}\text { Témoin } \\
0,02 \\
0,03 \\
0,04 \\
0,05 \\
0,07 \\
0,10\end{array}$ & $\begin{array}{r}0 / 20 \\
1 / 20 \\
6 / 20 \\
11 / 20 \\
14 / 20 \\
19 / 20 \\
20 / 20\end{array}$ & $\begin{array}{r}0 \\
5 \\
30 \\
55 \\
70 \\
95 \\
100\end{array}$ & $\begin{array}{c}\text { Temoin } \\
0,13 \\
0,18 \\
0,35 \\
0,50 \\
0,78 \\
0,89\end{array}$ & $\begin{array}{l}0 / 30 \\
1 / 30 \\
6 / 30 \\
15 / 30 \\
21 / 30 \\
27 / 30 \\
30 / 30\end{array}$ & $\begin{array}{c}0 \\
3,3 \\
20 \\
50 \\
70 \\
90 \\
100\end{array}$ \\
\hline
\end{tabular}

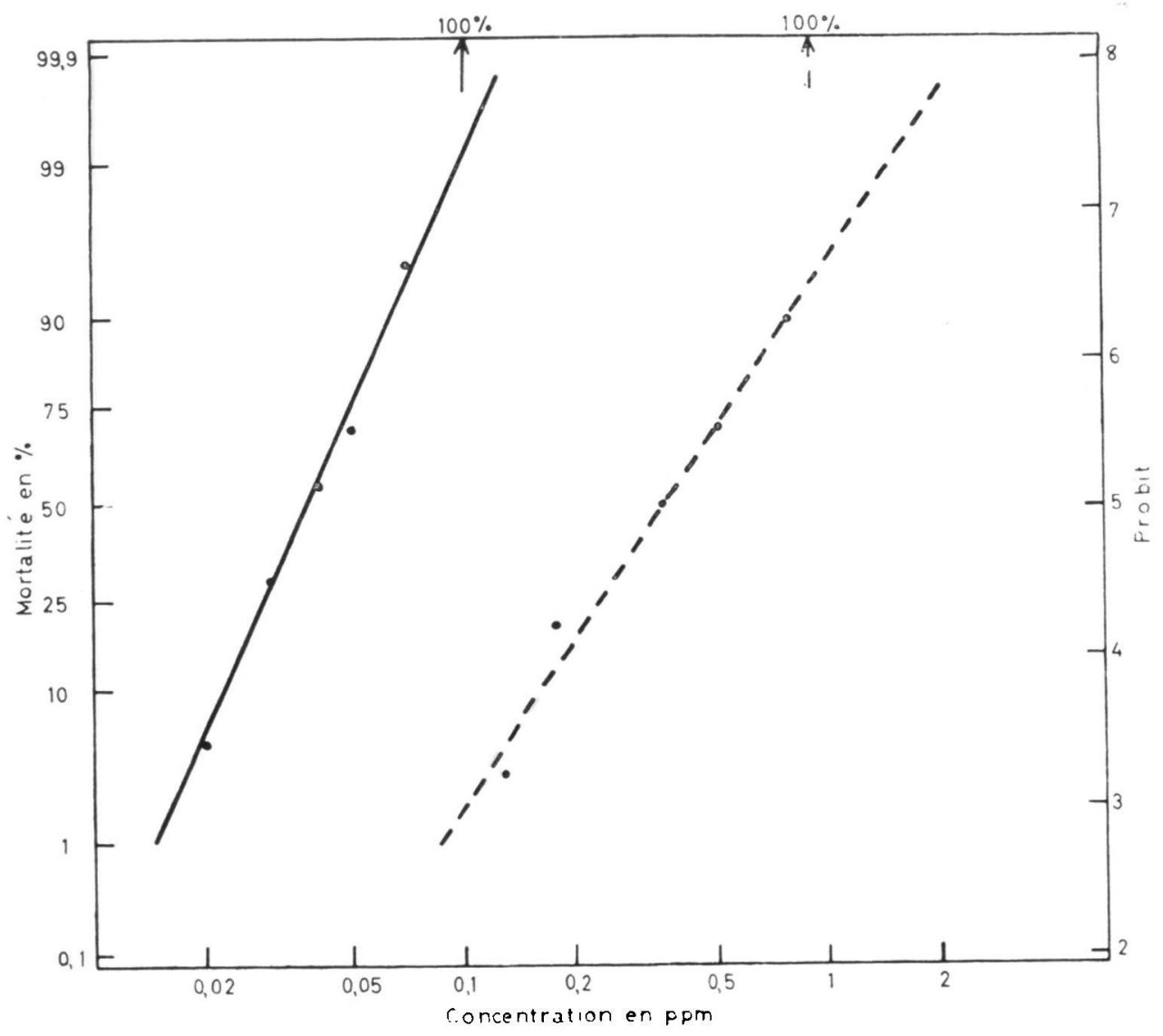

Les doses létales (DL) à $50 \%$ et à $90 \%$ de mortalitè sont facilement ètablies grâce à la droite de régression; nous les indiquons en ppm (part par 
million). On obtient pour le Bayluscide : $\mathrm{DL}_{\bar{j}}=0,037 \mathrm{ppm}$ et $\mathrm{DL}_{90}=0,063 \mathrm{ppm}$, puis pour le Frescon : $\mathrm{DL}_{50}=0,36 \mathrm{ppm}$ et $\mathrm{DL}_{90}=0,78 \mathrm{ppm}$.

\section{3 - Action sur poissons}

Des essais effectués sur le rotengle (Scardinius erythrophtalmus L.) provenant des étangs de la Dombes (1) ont permis de constater que les doses toxiques à $90 \%$ de mortalité étaient plus élevées avec le Bayluscide $\left(D L_{3 n}=0,25\right)$ qu'avec le Frescon $\left(D_{90}=0,12\right)$.

\section{4 - Discussion} du lac Léman.

Le Bayluscide est dix fois plus actif que le Frescon sur les moules

Le Frescon est deux fois plus actif que le Bayluscide sur les rotengles de la Dombes.

D'autre part, le Bayluscide est quatre fois plus toxique pour les moules que pour les rotengles, alors que le Frescon est six fois plus toxique pour les rotengles que pour les moules.

Si l'on compare l'action de ces produits sur les Gastéropodes vecteurs de la Bilharziose (Australorbis glabratus principalement) (PAULINI, CHAIA, DE FREITAS, 1961 ; RITCHIE, BERRIOS-DURAN, 1969) et sur la moule d'eau douce, on constate que cette moule est un peu plus sensible au Bayluscide, mai's nettement moins sensible au Frescon.

II est intéressant d'indiquer que le Bayluscide agit sur les Mollusques en freinant l'absorption d'oxygène, ce qui est vraisemblablement la cause de leur mortalité.

\section{4 - TOXICITE GENERALE DU BAYLUSCIDE ET DU FRESCON}

\section{1 - Bayluscide}

A la suite des recherches réalisées principalement par HECHT et GLOXHUBER (1962), GONNERT (1962), DESCHIFNS et FLOCH (1968), on peut résumer l'action de ce produit.

Le Bayluscide n'est pratiquement pas toxique pour les Mammifères et l'Homme. Des essais sur rats, souris et lapins par absorption buccale du produit mélangé à la nourriture ont permis de constater que les animaux supportaient aisément des doses très élevées $(236 \mathrm{~g}$ de Bayluscide en une année pour des chiens de $9 \mathrm{~kg} ; 75 \mathrm{~g}$ de Bayluscide en une année pour des rats de $175 \mathrm{~g}$ ). On a également constaté que l'Homme absorbait sans difficultès $30 \mathrm{mg} / \mathrm{kg}$ (soit $2 \mathrm{~g}$ pour un homme de $70 \mathrm{~kg}$ ). Chez les Mammifères, il y a métabolisation du Bayluscide en quelques heures et élimination du produit résorbé en 24 heures.

Les veitébrés à sang froid sont sensibles au Bayluscide (Batraciens, Poissons); c'est ainsi que le poisson Lebistes reticulatus supporte $0,3 \mathrm{ppm}$ de produit mais est tué par $1 \mathrm{ppm}$.

(1) Nous remercions Monsieur BELL, directeur de la Coopérative Piscicole de la Dombes, qui nous a procuré ces poissons 
Les organismes vivant dans le plancton sont également sensibles au Bayluscide, mais on a constaté que les populations se reconstituent rapidement.

Les Insectes aquatiques et leurs larves supportent des concentrations plusieurs fois plus élevées que celles qui sont nécessaires pour la destruction des Mollusques.

Les plantes aquatiques ne supportent pas la dose de 3ppm, mais, aux doses utilisées pour les Mollusques, on ne constate pas de dégâts ou tout au plus un flétrissement passager. Les plantes terrestres sont plus résistantes que les plantes aquatiques.

\section{2 - Frescon}

A la suite des travaux de plusieurs chercheurs, en particulier SHIFF, CROSSLAND el MILLAR (1967), on peut résumer l'action de ce produit.

A l'égard des Mammifères, des essais sur rats et souris prouvent que des doses de $10000 \mathrm{ppm}$ de Frescon dans la ration alimentaire sont supportées durant plus de trois mois; par utilisation du Carbone 14, on a constaté que la majeure partie des produits métaboliques du Frescon sont éliminés (65\% dans les fèces, $25 \%$ dans l'urine, 1,5 à $5 \%$ demeure dans le corps.

A l'égard des Poissons, la marge de sélectivité demeure étroite, comme l'ont prouvé des essais sur les Tilapia en Rhodésie (Tilapia mossambica est tuée à $50 \%$ par une concentration de $0,1 \mathrm{ppm}$ ). Le Frescon n'est pas toxique pour les Invertébrés aquatiques à la dose de $0,2 \mathrm{ppm}$ (essais effectués en Rhodésie).

Les Végétaux n'ont subi aucun effet phytotoxique lors des essais en laboratoire ou sur le terrain alors que les doses utilisées étaient dix fois supérieures à celles employées pour détruire les Mollusques.

\section{5 - INDICATIONS PRATIQUES}

A l'égard de la moule d'eau douce, le Bayluscide a une activité molluscicide plus grande que celle du Frescon; en outre, le Bayluscide est moins toxique pour les Poissons que le Frescon; il semble donc préférable d'utiliser le Bayluscide dans la lutte contre Dreissena polymorpha. Le Bayluscide est, par ailleurs, un produit peu cher.

\section{1 - Eaux piscicoles}

II est nécessaire d'éviter une concentration du Bayluscide qui puisse être toxique pour les Poissons.

II serait utile d'effectuer de nouveaux essais sur divers Poissons de nos eaux douces, mais, d'après les résultats déjà obtenus sur Scardinius et Lebistes, il semble que des doses de $0.1 \mathrm{ppm}$ seraient supportées par les Poissons.

Le Bayluscide peut être utilisé à partir de la poudre mouillable pour préparer des solutions à $5 \%$ de produit actif; ce sont de telles solutions qu'il faudrait verser dans les eai!x piscicoles en veillant à un mélange rapide avec l'eau à traiter.

Le Bayluscide peut être également utilisé à partir d'une préparation réalisée par GOODRICH (USA), (CARDARELLI, 1968); il s'agit d'une * disso- 
lution * du Bayluscide dans une matrice élastique de Néoprène. L'avantage de cette préparation est d'assurer une dissolution lente du Bayluscide dans l'eau à traiter car les molécules de Bayluscide ne sont libérées que lentement des molécules de Néoprène entre lesquelles elles sont insérées et auxquelles elles adhèrent. Une étude de contrôle a permis de constater que le Bayluscide-Néoprène, maintenu dans leau à traiter, continue à émettre le molluscicide durant une période de plusieurs mois, et mème d'années, à un taux fatal pour les mollusques mais non pour l'homme, les poissons et divers animaux du milieu aquatique. C'est sous la forme de pastilles ou de feuilles que l'on peut employer le Bayluscide-Néoprène. En tenant compte du volume d'eau et, s'il y a lieu, du courant, on peut apprécier la quantité de produit ainsi préparé qu'il serait nécessaire d'utiliser.

\section{2 - Canalisations d'alimentation en eau}

Si l'on peut interrompre deux fois par an durant 24 heures l'utilisation de la canalisation, il suffirait de remplir cette canalisation de solution entre 0,1 et $0,3 \mathrm{ppm}$ selon le $\mathrm{pH}$ de l'eau et de l'y maintenir durant 24 heures. Les dates à choisir sont en relation avec la biologie de la moule d'eau douce, c'est-à-dire d'abord après la fin de la période d'éclosion des larves (généralement fin de l'automne), puis en février ou mars pour tuer les jeunes moules qui auraient réussi à pénétrer après la période automnale.

$\mathrm{Si}$ l'on ne peut pas interrompre l'utilisation de la canalisation, il y aurait lieu de mélanger le Bayluscide à l'eau de la canalisation afin de maintenir une concentration de 0,1 à $0,3 \mathrm{ppm}$ durant 24 heures et ceci deux fois par an. II n'y aurait aucune crainte de toxicité pour l'Homme et les Mammifères utilisant cette eau, comme l'ont prouvé les recherches indiquées plus haut.

\section{6 - BIBLIOGRAPHIE SOMMAIRE}

CARDARELLI N. F., 1968. Method for dispersing toxicants for kill disease-spreading water-spawned larva. Trematodes, Molluscs and similar organisms, and products used in such method. United States Patent Office, 17 déc. 1968, $8 \mathrm{p}$.

DESCHIENS R. et FLOCH H.A., 1968. Action biologique comparée de 6 molluscicides dans le cadre de la prophylaxie des Bitharzioses. Conclusions pratiques. Bull. Soc. Path. Exot., 61, p. 640-650.

GÖNNERT R., 1962. Bayluscid, un nouveau produit pour la destruction des mollusques d'eau douce, d'importance médicale. Pflanz. Nach. Bayer, 15 , p. $4-25$.

GREENSHIELDS F. and RIDLEY J. E., 1957. Some researches on the control of Mussels in Water Pipes. J. Inst. Wat. Engin, 11, 3, p. 300-306.

HECHT G. et GLOXHUBER Ch., 1962. Recherches sur la tolérance de la 5,2' dichloro-4'- nitrosalicylanilide - éthanolamine (Bayluscid). Pflanz. Nachr. Bayer, 15, p. 34-41.

HOESTLANDT H., 1968. Rapport de Mission sur le problème du Mollusque Dreissena polymorpha dans l'approvisionnement en eau potable de la région d'Istambul. Org. mond. Santé, $25 \mathrm{p}$.

HOESTLANDT $H ., 1969$. Mussel infestation of water installations. WHO Chronicle, 23, 2, p. 84-86. 
HOESTLANDT $H$., 1971. Action de deux molluscicides (Bayluscide et Frescon) sur la moule d'eau douce, Dreissena polymorpha PALLAS. C. R. Acad. Sci. Paris, 272, p. $1912-1914$.

HOESTLANDT $H$. et LASSABLIEPE J., 1959. Destruction thermique de la moule d'eau douce. L'Eau, 46, 11, p. 38-41.

KINKELIN P. de, TUFFERY G. LEYNAUD G. et ARRIGNON J., 1968 a. Répartition géographique et interspecifique de la Bucéphalose à Bucephalus polymorphus (Baer 1827) (Trématode Bucephalidae) dans le peuplement piscicole du bassin de la Seine. C.R. Acad. Sci. Paris, 266, p.1 033-1 035.

KINKELIN P. de, TUFFERY G., LEYNAUD G. et ARRIGNON J., 1968 b. Etude épizootiologique de la Bucépralose larvaire à Bucephalus polymorphus (Baer 1827) dans le peuplement piscicole du bassin de la Seine. Rech. Vétér. 1, p. 77-98.

LITCHFIELD J.T. and WILCOXON F., 1949. A simplified method of evaluating dose-effect experiments. J. Pharmac exp. Ther., 96, p. 99-113.

PAULINI E., CHAIA G. and DE FREITAS J.R., 1961. Trials with the Moliuscicids Rhodiacid and Bayer 73. Bull. Org. mond. Santé, 25, p. 706-709.

RITCHIE L.S. and BERRIOS-DURAN L. A., 1969. Chemical Stability of molluscicidal compounds in water. Bul! Org. mond. Santé, 40, p. 471-473.

SHIFF C. J., CROSSLAND N.O. and MILLAR D.R., 1967. The Susceptibilities of Various Species of Fish to the Molluscicid N-Tritylmorpholine. Bull. Org. mond. Santé, 36, p. 500-507.

Summary of Information Frescon, 1968 Shell Chemicals, $16 \mathrm{p}$. 